\title{
Bioéticas latinoamericanas, nutrición clínica y pandemia por COVID-19
}

\author{
Latin American bioethics, clinical nutrition and COVID-19 pandemic \\ Bioética Latino-Americana, Nutrição Clínica e Pandemia por COVID-19
}

\author{
Camilo Manchola-Castillo ${ }^{1 *}$. \\ Recibido: 16 de junio de 2021. Aceptado para publicación: 3 de agosto de 2021. \\ https://doi.org/10.35454/rncm.v4supl1.312
}

\section{Resumen}

Objetivo: establecer una relación entre las bioéticas latinoamericanas (BLA) y la nutrición clínica, que permita a esta última enriquecer su reflexión mediante la incorporación de categorías de reflexión moral que le hagan responder de mejor manera a los desafíos colocados por la actual pandemia.

Material y métodos: investigación cualitativa a partir de un paradigma hermenéutico, reflexivo, sociocrítico y analítico dividida en tres momentos metodológicos: breve revisión de la nutrición clínica; construcción de un cuerpo categorial para las BLA; y establecimiento de una relación entre BLA y nutrición clínica.

Resultados y discusión: tres categorías de las BLA enriquecen definitivamente la nutrición clínica (derechos humanos, compromiso social y respeto por la vulnerabilidad humana), al proveer herramientas para que los expertos en nutrición clínica defiendan decisiones morales más justas.

Conclusiones: las BLA proporcionan marcos de referencia apropiados a la nutrición clínica y una aproximación entre ambas puede enriquecer las bases epistemológicas de la última.

Palabras clave: bioética, nutrición clínica, pandemia por COVID-19.

\section{Abstract}

Objective: To establish a relationship between bioethics in Latin America (BLA) and clinical nutrition (NC), in order to allow the latter to enrich its thinking by incorporating categories of moral reflection and enhance its ability to respond to the challenges posed by the current pandemic.

Material and methods: Qualitative research based on a hermeneutical, reflective, socio-critical and analytical paradigm, divided into three methodological moments: brief review of NC; construction of a categorical body for BLA; and establishing a relationship between BLA and NC.

Results and discussion: three categories of BLA definitely enrich NC (human rights, social commitment, respect for human vulnerability), by providing experts in clinical nutrition with tools to defend more righteous moral decisions.

Conclusions: BLA provide appropriate frames of reference to NC, and an approximation between the two can enrich the epistemological bases of the latter.

Keywords: Bioethics; Clinical Nutrition; COVID-19 Pandemic.

\section{Resumo}

Objetivo: estabelecer uma relação entre as bioéticas latino-americanas (BLA) e a nutrição clínica (NC), que permita a esta última enriquecer sua reflexão ao incorporar categorias de reflexão moral que the permitam responder de melhor maneira aos desafios colocados pela atual pandemia.

Material e métodos: pesquisa qualitativa baseada em um paradigma hermenêutico, reflexivo, sociocrítico e analítico, dividida em três momentos metodológicos: breve revisão da NC; construção de um corpo categórico para as BLA; e estabelecimento de uma relação entre BLA e NC.

Resultados e discussão: três categorias das BLA definitivamente enriquecem a CN (direitos humanos, compromisso social, respeito à vulnerabilidade humana), ao fornecer ferramentas para especialistas em nutrição clínica defenderem decisões morais mais justas.

Conclusões: os BLA fornecem marcos de referência adequados para a NC e uma aproximação entre ambas pode enriquecer as bases epistemológicas da última.

Palavras-chave: bioética, nutrição clínica, pandemia por COVID-19.
Universidad de Brasilia. Brasilia, Distrito Federal, Brasil.
${ }^{*}$ Correspondencia: Camilo Manchola-Castillo. camilomanchola@gmail.com 


\section{INTRODUCCIÓN}

Uno de los efectos que ha tenido la pandemia relacionada con la COVID-19 es que cada vez más personas han tenido que depararse con conflictos morales que antes competían exclusivamente a unos pocos individuos, y estos conflictos en variados casos se relacionan con el área de la nutrición clínica ${ }^{(1)}$. Así, la pandemia ha puesto en discusión especialmente el cuidado nutricional de grandes grupos humanos, sea por la desnutrición asociada a la enfermedad (en casos de hospitalizaciones masivas por COVID-19 en unidades de cuidados intensivos [UCI], por ejemplo), por una ingesta dietética menor a lo recomendado (en situaciones en las que las personas, por los devastadores efectos de la pandemia, pasan hambre, por ejemplo), o por ingesta dietética que conduce al sobrepeso y obesidad (en casos en los que, por las restricciones a la movilidad causadas por la pandemia, el gasto energético disminuye o la obtención de fuentes nutricionales variadas se ve comprometida).

Como pocas veces en la historia reciente, hoy se revela la enorme importancia del área de la nutrición clínica para las sociedades. De esta manera, actores, como los expertos en nutrición clínica, deben tomar puestos relevantes en la esfera nacional e internacional en órganos del gobierno decisivos para el manejo de las graves consecuencias nutricionales de la pandemia ${ }^{(1)}$, así como en el trabajo con las comunidades, acompanándolas para que se alimenten de una mejor manera y tomen mejores decisiones relativas a situaciones límite que la pandemia ha creado en términos nutricionales. En especial, el accionar de estos actores se revela especialmente relevante en la construcción de políticas en salud pública relacionadas con una alimentación adecuada y con la atención de condiciones de desnutrición relacionadas a la enfermedad, entre otras. En este sentido, la pandemia ha mostrado claramente el papel ético y moral que expertos en nutrición clínica cumplen en la realidad de países, comunidades y organizaciones internacionales ${ }^{(2)}$.

En este sentido, debe decirse que la pandemia ha mostrado a las sociedades de todos los países, como pocas veces, los enormes impactos que la desigualdad en el acceso a una alimentación adecuada tiene sobre el bienestar de los seres humanos. Así, decididamente ha desnudado que la inequidad y la desigualdad alimentaria que forman parte de regiones como América Latina tienen un papel fundamental en las condiciones de salud y enfermedades de las poblaciones que viven en estas regiones, especialmente en la forma como la
COVID-19 ha afectado a poblaciones especialmente vulnerables ${ }^{(3)}$. De esta manera, cada vez queda más claro que invertir tiempo y recursos en el área de la nutrición clínica es esencial, pues para enfrentar problemas como los que la pandemia mencionada ha generado es importante que la reflexión en esta área se perfeccione y progrese. Las circunstancias producidas por este fenómeno han dejado claro que la nutrición clínica, un área eminentemente inter- y transdiscipli$\operatorname{nar}^{(4)}$, necesita hoy más que nunca de elementos nuevos que le permitan responder de una mejor manera a los desafíos que encara.

El presente artículo propone establecer una relación entre las bioéticas latinoamericanas (BLA) y la nutrición clínica, que permita a esta última enriquecer su reflexión mediante la incorporación de categorías de análisis moral que le hagan responder de mejor manera a los desafíos relativos al acceso de grupos poblacionales a una alimentación adecuada, así como a conflictos morales relacionados con situaciones límite como las que experimentan quienes están en UCI por causa de la COVID-19; y busca hacerlo desde un enfoque bioético latinoamericano ${ }^{(5)}$ que defiende los derechos humanos, tiene compromiso social y está pautado en el respeto por la vulnerabilidad humana. El objetivo final es, pues, que el establecimiento de esa relación, a partir de un paradigma hermenéutico, reflexivo, sociocrítico y analítico revele elementos de las BLA que pueden contribuir a una mejor toma de decisiones por parte de especialistas en nutrición clínica en la actual coyuntura.

\section{ANTECEDENTES}

\section{La nutrición clínica}

La nutrición clínica es una ciencia básica interdisciplinaria y aplicada que se ocupa de las personas que necesitan cuidados nutricionales por desnutrición asociada a enfermedad o ingesta dietética, o por sobrepeso y obesidad. Su objetivo es aplicar los principios del cuidado nutricional, dentro de los que se encuentran, por ejemplo, la nutrición administrada artificialmente para garantizar un equilibrio en el estado nutricional, y para modular otras funciones biológicas, lo que impacta positivamente en el equilibrio del individuo ${ }^{(4)}$.

En una proporción importante, la nutrición clínica se lleva a cabo con pacientes hospitalizados; sin embargo, con más y más frecuencia la nutrición clínica lidia con pacientes ambulatorios, una vez que su área de actuación tiene que ver con variadas condiciones que 
no necesariamente remiten a ambientes hospitalarios, incluyendo contextos sociales diversos. Así, esta ciencia está cada vez más presente en la vida de las personas.

Incorpora diversos campos y tiene como objetivo mantener un equilibrio energético saludable en los pacientes, mientras intenta garantizar el aporte de cantidades suficientes de nutrientes como proteínas, vitaminas, minerales. Puede decirse, en este sentido, que la nutrición clínica se ocupa de la prevención, el diagnóstico y el manejo de los cambios nutricionales y metabólicos relacionados con enfermedades agudas y crónicas, y afecciones causadas por la falta o exceso de energía y nutrientes ${ }^{(5)}$.

Entonces, implica estudiar qué nutrientes son necesarios para que el cuerpo humano funcione y cómo lo que los individuos comen afecta su salud. De esta forma, esta ciencia analiza si una persona está consumiendo una cantidad adecuada de nutrientes para una buena salud y se preocupa por cómo el cuerpo procesa, almacena y desecha los nutrientes de los alimentos, además de cómo lo que cada individuo come afecta su bienestar general.

La nutrición clínica evalúa necesidades nutricionales basándose en el historial médico y familiar del paciente, su estilo de vida y pruebas de laboratorio para poder hacer recomendaciones sobre su dieta y necesidades nutricionales individuales. Así, brinda consejos sobre cambios en la dieta que pueden ayudar a prevenir enfermedades. Por eso, la nutrición clínica no es exclusiva de UCI, sino que actúa en una variedad de entornos, incluidos hospitales, agencias gubernamentales, compañías farmacéuticas, compañías de alimentos, entre otros.

También puede implicar el uso de suplementos dietéticos y nutricionales como una forma de mejorar la salud y prevenir enfermedades; de hecho, esto es quizás lo que más la caracteriza a los ojos de las personas del común. De esta forma, la nutrición clínica involucra el estudio de la relación entre los alimentos ingeridos y el bienestar del organismo, identificando los nutrientes y cómo se digieren, absorben, transportan, metabolizan, almacenan y utilizan.

La nutrición clínica se ha convertido en una práctica que se incorpora cada vez más al tratamiento médico convencional, pues puede mejorar el bienestar de un paciente en diversos momentos, dentro de los cuales se puede no solo destacar cuando se somete a protocolos farmacéuticos convencionales, sino también cuando se incluye en programas para prevenir enfermedades. En este sentido, es cada vez más claro que las necesidades nutricionales de un individuo son específicas.
Pero la nutrición clínica tiene una dimensión cada vez más social y política que debe ser mencionada y que se conecta con que es un campo eminentemente inter- $y$ transdisciplinar. $\mathrm{Al}$ respecto, debe mencionarse que esta se interesa en cómo el medio ambiente afecta la calidad y seguridad de los alimentos y cómo estos factores influyen en la salud y las enfermedades de los individuos; por este medio, defiende que se considere a la atención nutricional como un derecho humano en conexión con derechos de primera, segunda y tercera generación ${ }^{(6)}$.

Con respecto al enfoque basado en los derechos humanos, la nutrición clínica propone que la atención nutricional como derecho humano permite priorizar la nutrición y, de esa manera, luchar contra la malnutrición, defendiendo una atención nutricional óptima para todos. Así pues, la nutrición clínica propende por un desarrollo científico, social, educativo y económico que valore el cuidado nutricional y promueva el empoderamiento del paciente.

Queda claro que la nutrición clínica, lejos de ser una ciencia exclusiva de las UCI o de los pacientes gravemente enfermos, es un campo inter- $y$ transdisciplinar que espera aportar al cuidado nutricional de diversos individuos, y que defiende que responsables políticos y gestores institucionales creen políticas públicas que contribuyan a asegurar que exista una atención nutricional óptima para todos los individuos.

En ese sentido, la nutrición clínica defiende una agenda que aboga por la dignidad, la equidad y la igualdad entre los individuos, pues es consciente de que solo a través de ellas será posible la realización del derecho a la atención nutricional, una responsabilidad que claramente atribuye a los Estados, que deben crear políticas y programas que garanticen este derecho a todos los ciudadanos, independientemente de su origen económico, social, cultural, religioso o político ${ }^{(7)}$.

\section{Las bioéticas latinoamericanas}

La bioética fue creada en Estados Unidos hace aproximadamente medio siglo, en la década de 1970, para responder a los potenciales conflictos morales que los avances tecnocientíficos producían en relación con la salud humana y el medio ambiente ${ }^{(8)}$. Ese énfasis tecnocientífico de la bioética sería desafiado decididamente con la llegada de la bioética a América Latina en la década de 1990: las llamadas bioéticas latinoamericanas (BLA) propusieron una nueva territorialización epistemológica de la disciplina, incorporando temas sociales y defendiendo que los conflictos morales no 
solo son relativos a avances tecnocientíficos, sino a condiciones sociales y económicas que hacen que grandes grupos poblacionales sean vulnerados sistemáticamente en sus derechos fundamentales. De esta manera, las BLA propusieron la creación de categorías como situaciones emergentes (relacionadas con avances tecnocientíficos) y situaciones persistentes (relacionadas con vulneraciones de derechos fundamentales como el acceso a la salud, la educación y la alimentación) $)^{(9,10)}$.

Las BLA establecieron una conexión definitiva con los derechos humanos desde sus inicios, y eso queda muy claro dado que las BLA más reconocidas nacieron luego de la caída de dictaduras en Argentina y Brasil, claros ejemplos del irrespeto a los derechos humanos. Así, estas no solo reconocen como objeto de estudio situaciones como los dilemas relativos al uso de células madre, investigación con embriones, reproducción asistida, clonación y manipulación genética, sino falta de acceso a salud y mercantilización de la misma, desigualdad e inequidad, concentración de la riqueza, distribución de recursos, entre otros ${ }^{(11,12)}$.

Así pues, las BLA conceden un papel central a aquellos que sufren: discriminados y vulnerables, quienes son históricamente excluidos de los avances que ha dejado el proceso civilizatorio de la humanidad; al mismo tiempo, proponen la defensa, emancipación y liberación de estas personas por medio de procesos de intervención que les permitan participar, decidiry exigir que los Estados creen políticas y programas que garanticen los derechos fundamentales de los individuos. $\mathrm{Al}$ hacerlo, las BLA también dan un protagonismo decidido al Estado, no como detentor de legitimidad para decidir sobre conflictos morales, sino como garante de condiciones justas para todos los individuos.

Entre sus herramientas conceptuales y metodológicas, las BLA presentan tres categorías teóricas que pueden ser de utilidad para la toma de decisiones en nutrición clínica en el contexto actual. La primera ya fue mencionada y es relativa a los derechos humanos. Las BLA argumentan que en el mundo actual no es éticamente defendible la existencia de vidas humanas que valgan más que otras, tomando como base el color de la piel, el estrato socioeconómico, la condición médica, la orientación sexual, el género que la sociedad les ha atribuido o el sexo biológico con el que nacieron; de otra manera, los cuerpos que habitan ${ }^{(13)}$. Por lo tanto, las BLA, basándose en los derechos humanos, defienden que todos los seres humanos deben ser tratados con equidad e igualdad, pues todos son igualmente dignos $^{(14)}$. No hay espacio para que solo algunos pocos tengan acceso a alimentación, educación o salud; esta es una reivindicación que también es hecha por la Unesco a través de la Declaración Universal sobre Bioética y Derechos Humanos ${ }^{(15)}$.

Específicamente sobre el asunto de la dignidad humana, elemento central de los derechos humanos, las BLA han aportado para una comprensión contextual de este elemento, defendiendo que la dignidad, si bien es innata a cada ser humano, depende del reconocimiento que los otros seres humanos hagan de ella, y también es dependiente de un medio ambiente seguro y saludable. De esa manera, las BLA proponen un entendimiento de la dignidad humana que se aleje del mero antropocentrismo e individualismo al que se le ha históricamente asociado, acercándose a una visión más holística, incluyente y relacionada con el medio ambiente.

La segunda es el compromiso social ${ }^{(10)}$. Esta expresa la necesidad de promover una bioética politizada que haga frente a las constantes injusticias sociales y económicas a las que son sometidos diversos individuos. Las BLA rechazan la existencia de una bioética neutra, exclusivamente académica y alejada de las luchas sociales de los seres a los cuales debe servir, y abogan por una reflexión bioética que rechace, por ejemplo, la imposición, por parte de países ricos, de estándares morales para la conducción de investigación clínica en países pobres (realización de ensayos clínicos usando placebo y no garantizando el acceso posestudio a medicamentos) o la concentración de los beneficios de la investigación y el desarrollo científico solo en los países ricos, cuando para su alcance han sido centrales los países pobres, que en muchas ocasiones financian a través de la transferencia de dividendos de multinacionales o involucran a sus obligaciones en diversos protocolos científicos.

La tercera es el respeto por la vulnerabilidad humana, que defiende que para la deliberación moral es esencial considerar la vulnerabilidad social de diversos individuos. De esta manera, las BLA abogan por que la clásica visión de la vulnerabilidad humana apenas conectada a condiciones de salud, género o edad sea ampliada e involucre marcos contextuales en los que sean tomadas en cuenta nociones de historicidad social, política y económica. Las BLA argumentan, de esta manera, que existe un territorio epistémico necesariamente contextual e histórico que debe ser entendido para comprender la vulnerabilidad humana y, al hacerlo, revelan la importancia que tienen los factores sociales en la toma de decisiones morales ${ }^{(16)}$. 


\section{La bioética y la nutrición clínica}

Existen diversos trabajos que discuten la relación entre bioética y nutrición clínica ${ }^{(6)}$ por la vocación multi-, inter- y transdisciplinar de ambos campos, por la definitiva cercanía de la bioética a las cuestiones de la nutrición clínica y por las implicaciones éticas que a menudo tienen las decisiones que los expertos en nutrición clínica deben tomar, comúnmente relativas a individuos vistos como vulnerables. En ese sentido, la posibilidad de alimentar a un enfermo por vía artificial (uno de los grandes avances en la medicina del siglo $\mathrm{XX})$ ha traído cuestionamientos fundamentales que han sido abordados por la bioética, muy especialmente por la bioética tradicional estadounidense, pautada por cuatro principios: autonomía, beneficencia, no maleficencia y justicia.

Sin embargo, si existen esas conversaciones entre las dos áreas, ninguna de ellas ha aproximado las BLA, basadas en los derechos humanos (a diferencia de la bioética estadounidense), la justicia social y la vulnerabilidad humana a la nutrición clínica. Así, existe una oportunidad importante para acercar dos áreas que, aunque ya han sido aproximadas, no lo han hecho desde perspectivas latinoamericanas y tampoco a partir de aquello que la bioética puede ofrecer ante el enorme desafío que la COVID-19 ha significado para el mundo actual, en general, y el mundo del sur global, en particular.

\section{MATERIAL Y MÉTODOS}

Esta es una investigación cualitativa a partir de un paradigma hermenéutico, reflexivo, sociocrítico y analítico ${ }^{(17)}$. Está fundamentada en la búsqueda de un conocimiento que procure encontrar respuestas posibles a realidades que desafían el bienestar social y comunitario, de modo que sea viable transformarlas. En ese sentido, esta es una investigación orgánica y comprometida socialmente ${ }^{(18)}$. La investigación adopta un diseño metodológico coherente con el paradigma citado desde el objeto de estudio de la nutrición clínica, la lectura disciplinar de la realidad y el reforzamiento de los procesos bioéticos en sus diferentes dimensiones ${ }^{(19)}$.

Según los objetivos, tres momentos metodológicos orientan la implementación de la investigación: revisión a partir de fuentes secundarias (documentales), de la naturaleza, fines y niveles organizativo y operacional e impactos sociopolíticos de las diferentes expresiones de la nutrición clínica que han emergido; construcción de un cuerpo categorial en relación con las BLA, espe- cialmente sus categorías de derechos humanos, compromiso social y respeto por la vulnerabilidad humana; y establecimiento de una relación rigurosa, estructurada y contextual entre las BLA y la nutrición clínica.

\section{RESULTADOS Y DISCUSIÓN}

\section{Los derechos humanos defendidos por las BLA y la nutrición clínica en el contexto de la pandemia por COVID-19}

Los derechos humanos defendidos por las BLA permiten a los expertos en nutrición clínica defender moralmente el cuidado nutricional como un derecho humano ${ }^{(7)}$, especialmente en tiempos en los que la pandemia por la COVID-19 pone cada vez en mayor riesgo este cuidado. La nutrición clínica puede, con la ayuda de las BLA, cuestionar la moralidad que está detrás de la realidad que muestra que son exactamente los países más pobres aquellos que han sufrido las peores consecuencias en términos nutricionales de sus poblaciones. Más que eso, la noción de derechos humanos de las BLA puede permitir a expertos en nutrición clínica reclamar que esto no acontezca, pues conforme a principios acordados por todos los países en diversos documentos internacionales, todos los seres humanos son iguales y, por lo tanto, se debe velar por el aseguramiento de condiciones de vida dignas que involucran claramente el derecho a gozar de salud y calidad de vida, solo posible a través de un cuidado nutricional adecuado ${ }^{(7)}$.

La lectura contextual, holística e incluyente que proponen las BLA para el concepto de dignidad humana, elemento central de los derechos humanos, es importante para la nutrición clínica (cuyo propósito central es alimentar a la persona enferma), porque contribuye a la defensa de que la nutrición sea vista de una manera compleja y transdisciplinar, resaltando que depende de factores sociales, económicos, ambientales y políticos. En este sentido, la comprensión de la dignidad que tienen las BLA puede contribuir a que el área de la nutrición clínica argumente que es necesario, para proteger la dignidad de las personas, considerar, por ejemplo, las profundas desigualdades sociales existentes en el mundo.

\section{El compromiso social de las BLA y la nutrición clínica en el contexto de la pandemia por COVID-19}

Las BLA revelan, a través de su compromiso social, la necesidad de que los Estados creen programas y polí- 
ticas que aseguren el cuidado nutricional como derecho humano en momentos tan críticos como los que la pandemia ha producido. En este sentido, las BLA pueden contribuir a que los expertos en nutrición clínica aboguen por la importancia que tiene la proposición de acciones nacionales e internacionales que garanticen que las poblaciones más frágiles de países pobres tengan acceso a alimentos adecuados y, de esta manera, tengan mayor probabilidad de soportar las consecuencias de la infección por COVID-19 sin mayores complicaciones. A este respecto, hay diversos estudios que han demostrado esta conexión entre desigualdad social y mal pronóstico en la evolución de la enfermedad ${ }^{(20)}$. El compromiso social de las BLA permite a expertos en nutrición clínica exigir que las decisiones de todos los países se basen en la dignidad universal del ser humano, no importando su origen, religión, sexo, orientación sexual o raza.

\section{El respeto por la vulnerabilidad humana propuesto por las BLA y la nutrición clínica en el contexto de la pandemia por COVID-19}

La BLA muestran, por medio de su categoría de respeto por la vulnerabilidad humana, que es necesario contextualizar cualquier decisión en nutrición clínica, no solo tomando en cuenta las tradicionales consideraciones de la vulnerabilidad relativas al género o la edad, sino aspectos sociales y económicos ${ }^{(9,10)}$. En ese sentido, las BLA permiten que expertos en nutrición clínica defiendan la necesidad de que decisiones relativas a la priorización de recursos alimenticios y atención de enfermos de determinadas edades y condiciones de salud estén pautadas por directrices que consideren las desigualdades sociales y no apenas protocolos definidos previamente por agencias nacionales o internacionales ${ }^{(21,22)}$. Muestra de esto sería la defensa del cuidado nutricional como un derecho humano y no como un servicio, por parte de la nutrición clínica, y también su defensa de una atención nutricional prioritaria a determinadas poblaciones, vulneradas históricamente (Tabla 1).

Tabla 1. Relación entre las BLA y la nutrición clínica

\begin{tabular}{|l|}
\hline Categorías de las BLA que enriquecen la nutrición clínica \\
\hline Derechos humanos \\
\hline Compromiso social \\
\hline Respeto por la vulnerabilidad humana \\
\hline
\end{tabular}

Fuente: elaboración propia.

\section{CONSIDERACIONES FINALES}

Los casos de infección y las muertes por COVID-19 no han dejado de aumentar desde que el virus fue identificado en diciembre de 2019. En consecuencia, los impactos sociales y económicos han sido también numerosos, especialmente en regiones como América Latina, en donde la pandemia ha demostrado claramente que poblaciones particularmente pobres $y$ vulneradas socialmente son las que están siendo más afectadas, pues son aquellas que históricamente han visto sus derechos a una buena alimentación y a salud ser más irrespetados. De esta manera, es necesario defender enfoques teóricos del área de salud pública, en general, y del área de la nutrición clínica, en específico, que consideren estas inequidades y desigualdades en América Latina para que así se logre luchar contra ellas ${ }^{(23)}$.

En ese sentido, proponer marcos de referencia alternativos, como los que presentan las BLA, para enriquecer la práctica y reflexión de la nutrición clínica es esencial y es eso lo que esta investigación ha pretendido hacer. Específicamente, las BLA, a partir de las tres categorías propuestas en este trabajo, ofrecen una justificación moral a la nutrición clínica para que defienda la adopción de políticas y programas gubernamentales que promuevan la acción institucional del Estado y contribuyan a que las desigualdades nutricionales que hace mucho padecen diversos individuos, y que han sido empeoradas por la pandemia, sean superadas. Así, este artículo invita a que la nutrición clínica, un área tan transdisciplinar como las BLA, construya sobre el compromiso social y transdisciplinar de las BLA, su propio compromiso.

Finalmente, el establecer una relación entre BLA y nutrición clínica, a través de las categorías ya mencionadas, podría hacer que las políticas públicas en nutrición sean pensadas de forma diferente y, de esa manera, que el cuidado nutricional sea visto (y, especialmente, defendido y garantizado) como un derecho humano, enriqueciéndose así las bases epistemológicas de la nutrición clínica. Para este fin la Declaración Universal sobre Bioética y Derechos de la Unesco también puede ser muy útil(15).

\section{Financiación}

Este artículo fue financiado por la Coordinación de Perfeccionamiento de Personal de Educación Superior/ Ministerio de Educación/Brasil (Capes/MEC). 


\section{Conflicto de intereses}

Ninguno.

\section{Referencias bibliográficas}

1. Mayasari NR, Ho DKN, Lundy DJ, Skalny AV, Tinkov AA, Teng I-C, et al. Impacts of the COVID-19 Pandemic on Food Security and Diet-Related Lifestyle Behaviors: An Analytical Study of Google Trends-Based Query Volumes. Nutrients. 2020;12(10):3103. doi: 10.3390/nu12103103

2. Birgisdottir BE. Nutrition is key to global pandemic resilience. BMJ Nutr Prev Health. 2020;3(2):129-132. doi: 10.1136/ bmjnph-2020-000160

3. Cárdenas J, Robles-Rivera F, Martínez-Vallejo D. Élites empresariales y desigualdad en tiempos de pandemia en América Latina. Revista Española de Sociología. 2020;29(3): 715-26.

4. Cardenas D. What is clinical nutrition? Understanding the epistemological foundations of a new discipline. Clin Nutr ESPEN. 2016;11:e63-e66. doi: 10.1016/j.clnesp.2015.10.001.

5. Cederholm T, Barazzoni R, Austin P, Ballmer P, Biolo G, Bischoff SC, et al. ESPEN guidelines on definitions and terminology of clinical nutrition. Clin Nutr. 2017;36(1):49-64. doi: 10.1016/j.clnu.2016.09.004.

6. Allison SP. Basics in clinical nutrition: Ethical and legal aspects. Clin Nutr ESPEN. 2008;3(6):e298-e302. doi: 10.1016/j.eclnm.2008.07.004

7. Cardenas D, Bermudez C, Echeverri S. Is nutritional care a human right? Clin Nutr Exp. 2019;26:1-7. doi: 10.1016/j. yclnex.2019.05.002

8. Potter VR. Bioethics: Bridge to the future. New Jersey: Prentice-Hall Inc.; 1971.

9. Garrafa V, Porto D. Intervention Bioethics: A Proposal For Peripheral Countries in A Context of Power and Injustice. Bioethics. 2003;17(5-6):399-416. doi: 10.1111/14678519.00356

10. Garrafa V. Da bioética de princípios a uma bioética interventiva. Bioética. 2005;13(1):125-34.

11. Manchola CCH, Garrafa V, Cunha T, Hellmann F. El acceso a la salud como derecho humano en políticas internacionales: reflexiones críticas y desafíos contemporáneos. Ciênc. saúde coletiva. 2017;22(7):2151-2160. doi: 10.1590/141381232017227.04472017

12. Garrafa V, DA Cunha TR, Manchola C. Access to Healthcare: A Central Question within Brazilian Bioethics. Camb Q Healthc Ethics. 2018; 27(3):431-39. doi: 10.1017/ S0963180117000810

13. do Nascimento WF, Garrafa V. Por uma vida não colonizada: diálogo entre bioética de intervenção e colonialidade.
Saude soc. $2011 ; 20(2): 287-99$. doi: 10.1590/S010412902011000200003

14. Lander E. Ciencias Sociales: saberes coloniales y eurocéntricos. En: Lander E. La colonialidad del saber: eurocentrismo y ciencias sociales perspectivas latinoamericanas. Buenos Aires: CLACSO; 1993. p. 152.

15. Organización de las Naciones Unidas para la Educación, la Ciencia y la Cultura. Declaración Universal sobre Bioética y Derechos Humanos [Internet]. París: UNESCO, 2005. [Consultado el 11 de agosto de 2021]. Disponible en: http:// portal.unesco.org/es/ev.php-URL_ID $=31058 \& U R L$ $\mathrm{DO}=\mathrm{DO}_{-}$T OPIC\&URL_S E C T I O N $=201$. html\#: : text $=\mathrm{La} \% 20 \mathrm{Declaraci} \% \mathrm{C} 3 \%$ B 3 n $\% 20$ trata $\% 20$ de $\% 20$ las,dimensiones $\% 20$ sociales $\% 2$ C $\% 20$ jur\%C3\%ADdicas\%20y\%20ambientales

16. Feitosa SF, do Nascimento WF. A Bioética de Intervenção no contexto do pensamento latino-americano contemporâneo. Rev Bioét. 2015;23(2):277-84. doi: 10.1590/198380422015232066

17. Cebotarev EA. El enfoque crítico: una revisión de su historia, naturaleza y algunas aplicaciones. Rev Latinoam Cienc Soc Niñez Juv. 2003;1(1):1-27.

18. Fals BO, Rahman A. La situación actual y las perspectivas de la IAP en el mundo. En: Análisis Político. Instituto de Estudios Políticos y Relaciones Internacionales. Bogotá: Universidad Nacional de Colombia; 1999. p. 205-230.

19. Della PD, Keating M. Enfoques y Metodologías de las ciencias sociales. Una perspectiva pluralista. Revista Mad. 2014; 30:148-51.

20. Food and Agriculture Organization of the United Nations. Update on COVID-19 and its impact on food security and nutrition, and food systems [Internet]; 2020. [Consultado el 11 de agosto de 2021]. Disponible en: http://www.fao.org/3/ nd384en/nd384en.pdf

21. Rasmussen SA, Jamieson DJ. Public Health Decision Making during Covid-19. N Engl J Med. 2020;383(10):901-03. doi: 10.1056/NEJMp2026045

22. Martinez-Sanz J, Pérez-Molina JA, Moreno S, Zamora J, Serrano-Villar S. Understanding clinical decision-making during the COVID-19 pandemic. EClinicalMedicine. 2020;27:100539. doi: 10.1016/j.eclinm.2020.100539

23. Álvarez A, León D, Medellín M, Zambrano A, Zuleta H. El coronavirus en Colombia: vulnerabilidad y opciones de política [Internet]. Bogotá: PNUD América Latina y el Caribe, 2020. [Consultado el 11 de agosto de 2021]. Disponible en: https://www.latinamerica.undp.org/content/rblac/es/ home/library/crisis_prevention_and_recovery/el-coronavirus-en-colombia--vulnerabilidad-y-opciones-de-politic.html 